\title{
Individual differences and segment interactions in throwing *
}

\author{
Robert J. Neal and Conrad W. Snyder, Jr.
}

The University of Queensland, Brisbane, Australia

Pieter M. Kroonenberg

University of Leiden, Leiden, The Netherlands

\begin{abstract}
Neal, R.J., C.W. Snyder, Jr. and P.M. Kroonenberg, 1991. Individual differences and segment interactions in throwing. Human Movement Science 10, 653-676.

Arm segment velocities of 12 athletes throwing three differently weighted balls were analyzed by three-mode principal component analysis. Individual differences were characterized in terms of the combined influences of the phases of the throwing motion and the arm segment velocity relationships established in those phases. Using three individual differences components, three velocity measures components and four time phase components, $75 \%$ of the variance was described. The arm segment velocity relationships were described by two main components identified as directional velocity and proximal versus distal velocity. The time periods components distinguished between relationships among the arm segment velocities that occur in the windup versus those of the release phase. Three individual differences components are identified and appeared to be related to a general throwing style, the influence of skill level on technique, and the differential effect of the varying ball weights, respectively. Each athlete's throws are weighted combinations of these three components. The timing of segment involvement is investigated and the results indicate sequential patterns from proximal to distal as the throw unfolds. However, the results also suggest that different principles may apply to different throwers and that the summation of speed principle should not be applied universally to explain segment motion and interaction.
\end{abstract}

\section{Introduction}

When the intention of a movement is to generate maximum speed at the distal end of a kinematic linkage, for example, the action of

* Requests for reprints should be sent to R.J. Neal, Dept. of Human Movement Studies, The University of Queensland, Brisbane, Queensland 4072, Australia. 
throwing a ball, there may be a set order of body segment involvement to produce such an outcome. A number of authors (Bunn 1972; Dyson 1973; McCloy 1960) have proposed a kinetic link theory in which the sequential order of any movement for maximal speed begins with the large, strong, proximal muscles followed by the small, weak, distal muscles. Subjective interpretation of research results has provided evidence supportive of the kinetic link theory (e.g., Alexander and Haddow 1982; Ariel 1976; Davis and Blanksby 1976; Milburn 1982; Neal and Wilson 1985), but systematic, quantitative research which specifically tests the strength of the kinetic link theory has not been found.

Postulates regarding the summation of speed, force, and angular momentum in the sequencing of movement, all of which bear on the validity of the kinetic link theory, have been presented to account for specific empirical relationships between mechanical variables implicated by the theory. Early research on the contribution of individual segments to movement outcomes centered on the importance of isolated segments to the performance (Davis and Blanksby 1976; Hoshikawa and Toyoshima 1976; Toyoshima et al. 1974). Immobilization methods, in which isolated segments were systematically restrained, were used and the performance outcome was attributed to the involved segments. Inherent in these early studies was the assumption that each segment makes a contribution to motion that is independent of the other segments.

Later work centered on the timing of peak velocities of the involved segments. Milburn (1982) and Neal and Wilson (1985) demonstrated that peak angular velocity of the proximal segments preceded the peaks of the distal segments for the golf swing while Cavanagh and Landa (1976) presented similar results for a karate chop. Roberts et al. (1974) produced similar results for a soccer toe-kick and Hatze's (1976) findings for a controlled, target kick, too, were comparable. These studies support a summation of speed principle which states (Bunn 1972: 41) that when the movement of several members of the body are involved in developing optimum speed, the speed of each successive member should be faster than that of its predecessor, should start at the moment of greatest velocity of the preceding member, and be in the direction of the objective.

Ariel (1975), Koniar (1973), Kunz. (1974), and Plagenhoef (1971), in ain attempt to understand the timing of the peaks in velocity, investi- 
gated the acceleration time histories of the involved segments. Ariel (1975) and Plagenhoef (1971) contended that the correct sequence required a positive acceleration followed by a negative acceleration of the most proximal segment. The negative acceleration was believed to aid in positively accelerating the adjacent, distal segment. This pattern of positive followed by negative acceleration should continue outwardly to the most distal segment. In contrast, Koniar (1973) stated that the positive accelerations of the segments must peak simultaneously in order to produce maximum force. Koniar supported his hypothesis with data on vertical jumping. Work reported recently by Hudson (1986) also showed that for vertical jumping, a simultaneous timing of acceleration peaks was optimal. A possible reason for the disparate findings is that the tasks studied by Ariel (1975) and Plagenhoef (1971) and by Koniar (1973) and Hudson (1986) are very different. Ariel's (1975) theory pertained to throwing, in which a small, separate mass was thrown for maximum distance whil: the vertical jump task investigated by Koniar (1973) and Hudson (1986) involves the acceleration of a relatively large mass, the whole body. Thus, the summation of speed principle cannot be applied universally and must take into account the purpose and nature of the activity.

Putnam's (1980) results support this statement. She studied segment contributions in two activities, a punt kick and a gymnastic dismount. Results from her studies indicated that the summation of speed principle was supported by the data of the punt kick but violated for the data of the gymnastic dismount. This result is not unexpected since the gymnastic dismount would hardly benefit from the generation of maximum speed. It appears, therefore, that further investigation of segment interaction is warranted in order to understand segment interactions in movement. Three-mode component analysis was used to examine the interactions and time relationships among different arm segments in terms of velocity variance.

\section{Methods}

\section{Subjects}

Twelve right-handed, male, volunteer, university students formed the experimental group. All subjects signed an informed freedom of con- 
sent document prior to participation and wore only shorts and sporting shoes during testing.

\section{Protocol}

Subjects were filmed while performing six throws for maximal distance. Only data for one throw of each condition are presented here since extensive analysis (Neal 1988) demonstrated that the results from the second trial were extremely similar to those of the first. A Photosonics high speed cine camera located with its film plane parallel to and approximately $30 \mathrm{~m}$ from the assumed plane of motion of the throwers, and operating at 50 frames per second was used to film subjects. Actual framing rate was calculated from the timing marks on the edge of the film produced by a crystal controlled LED operating at $100 \mathrm{~Hz}$. A linear scale situated in the assumed plane of motion was filmed prior to filming the subjects. The data presented by Elliott et al. (1986) showed inat for baseball pitchers, $90 \%$ and $96 \%$ for the fast and curve balls respectively of the release velocity was accounted for by sagittal plane motion of the three major joints of the upper limb, the shouluer, elbow and wrist. In light of these data, it was considered appropriate to undertake a simple, planar analysis rather th'n go to the expense in terms of complexity and time, of a three-dimensional analysis.

Only one filming session was used and subjec'; completed all six trials at this time. In order to distribute the possibility of fatigue effects in the experiment, the order of throwing the differently weighted balls was varied across subjects. A Latin square design was used which incorporated four blocks. Subjects were randomly assigned to blocks and to an order of treatment, but equal numbers of subjects were assigned to the six possible orders of treatment.

Subjects were instructed to throw a ball for maximal distance using an overarm style. No run up was allowed but a step off the right foot onto the left foot was permitted. Subjects performed five trials of each condition of which only the last two were filmed. The masses of the three softballs were $180 \mathrm{~g}, 802 \mathrm{~g}$ and $1316 \mathrm{~g}$. Subject anthropometry was taken prior to filming.

\section{Data preprocessing}

The film was digitally analyzed using a PCD Motion Analyzer with digitizer. Four points, marking the segment endpoints of a 3-segment 
model of the arm, plus the ball were digitized for each film frame. The field of view was approximately $2.5 \times 2.0 \mathrm{~m}$ giving a spatial resolution, after digitizing and scaling, of $\pm 2 \mathrm{~mm}$. Every frame, commencing with the frame in which the left foot broke contact with the ground for the step and finishing with one frame past release, was digitized.

The three segments of the right upper limb, the arm, the forearm, and the hand, were modelled as a system of simply-linked rigid members. Dempster and Gaughran's (1967) segr.ent parameter data on segment mass and center of mass (CM) location were used in the model. A point mass, located at the ball's center, was used to model the ball. For all frames prior to release, the mass of the hand was considered to be the sum of the ball's mass and its own mass.

The position data of the segment CMs were smoothed using a 25-point, low-pass finite impulse response digital filter (McClellan et al. 1973). Before filtering, 12 extra data points were extrapolated to each cod of the data set by fitting a third order polynomial to the first or last 10 data points. This procedure allowed the whole data set to be smoothed, and did not arbitrarily force the second derivative at the endpoints to zero as does the extension procedure proposed by Lesh et al. (1979).

Because not all subjects completed the throw in the same period of time every trial was time normalized to $100 \%$ of the throwing cycle. An interpolative cubic spline (De Boor 1978) was used to complete the normalization process and all trials were represented by 30 points (see Neal (1988) for precise details;. The horizontal and vertical components of the CM velocities of the three segments were calculated using a first central difference algorithm. Since these data were components of the velocity vector and the camera to subject distance was large, there was little error in the velocity measures due to the assumptions made about sagittal plane motion.

\section{Three-mode principal component analysis}

The data from this study can be arranged in terms of three classification sets or modes: 6 upper limb segment velocity measures, 28 time periods, and 36 experimental units (12 subjects throwing 3 differently weighted balls). To describe the data in components in an insightful way it is necessary to reduce the larger, observed data set to a smaller, 
quantitative portrait of underlying relationships among the original data classification levels. The technique chosen to obtain such a portrait is three-mode principal component analysis. In such an analysis, each of the classification modes is assumed to be reducible to a new, smaller, and more fundamental 'derivative' set, such that there is a component set for the velocity measures of the segments, reflecting the influences of the arm segments in the action, another component set for time, reflecting the patterns of action across time, and a third component set for the experimental units, reflecting individual differences among the athletes and among their three tosses. These component sets describe the relationships within each of the original classification modes. The interactional information concerning the component sets is contained in a 'core' matrix. The core is the central feature of this methodology by which the large three-mode matrix of data is reduced to its fundamental variance components in a smaller three-mode core matrix. The core matrix is bounded and defined by the idealized velocity measures, time periods and experimental units. An entry in the core matrix represents the importance of a particular combination of .umponents from each of the three modes. Fig. 1 provides a diagram showing,

Data matrix

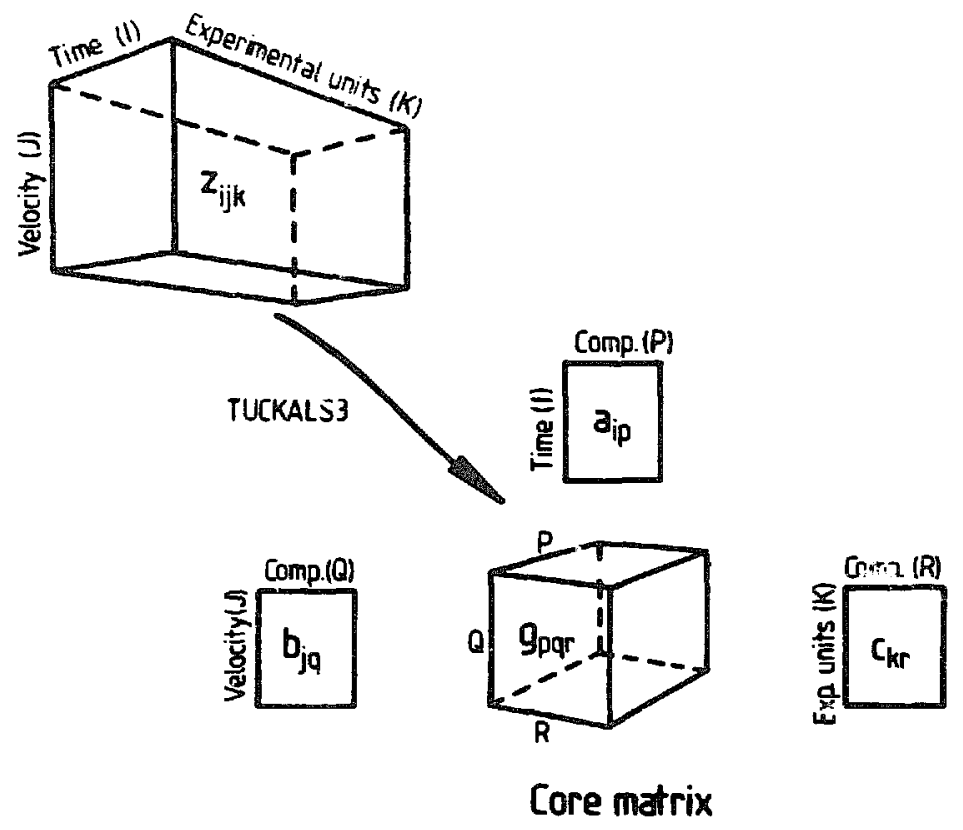

Fig. 1. Diagram illustrating the way in which the data of this study were arranged for the three-mode analysis. 
conceptually, how the data can be arre nged in a three-mode data box and how they are represented by the model.

Formally, the model is given by (see Tucker 1966):

$\hat{Z}_{i j k}=\sum_{p=1}^{P} \sum_{q=1}^{Q} \sum_{r=1}^{R} a_{i p} b_{j q} c_{k r} g_{p q r}$,

where, $\hat{Z}_{i j k}$ is the estimated velocity score of the $j$ th arm segment of the $k$ th experimental unit at the $i$ th time period, $a_{i p}$ is the score of the $i$ th time period on the $p$ th time period component, $b_{j q}$ is the score of the $j$ th velocity measure on the $q$ th velocity measure component, $c_{k r}$ is the score of the $k$ th experimental unit on the $r$ th individual differences component, and $g_{p q r}$ is a general element in the core matrix $\boldsymbol{G}$, reflecting the variance attributable to each combination of the $P, Q$, and $R$ components of the three modes of data. In general, the number of derived components, $P, Q$, and $R$, is less than their $I, J$, and $K$ associated observational sets and rarely greater than five, because most of the variance in a data set is contained within the first few components.

The method to solve the parameters of this three-mode model is an alternating least squares minimization approach to the approximate decomposition of the data box, and it is implemented in the computi: program TUCKALS3 (Kroonenberg and Brouwer 1985). Details of this algorithm are provided in Kroonenberg and De Leeuw (1980), and further information on the general approach is given by Kroonenberg $(1983,1984)$ and Snyder (1988).

Since the method yields least squares estimates of the parameters, the total sum of squares can be meaningfully decomposed, such that:

$$
\text { SS }(\text { DATA })=\text { SS }(\text { FIT })+\text { SS }(\text { RESIDUAL })
$$

and each of the individual mode solvtions and the core solution can be evaluated and interpreted in terms of contributions to the sum of squares of fit (for technical details see Ten Berge et al. 1987). Each coefficient of the mode components reflects the proportion of variance attributable to the derived solution, and the core entries summarize the total fit, patterned according to the interactional influences of the combined components across the three modes. 
Recause of scaling differences in these data, the measures were stanciardized within each experimental unit, the points in time were centered (means removed to reflect pattern variability only), and the units were normalized (variabilities equated so that all units would be characterized in the least squares solution). These preprocessing steps were carried out in order to improve the characteristics of the data for the least squares procedure (Harshman and Lundy 1984).

\section{Results and discussion}

The results and discussion center on description of the components of the original data set. Table 1 shows selected anthropometric measures of the subjects, and average distances thrown for each of the three differently weighted balls.

\section{Velocity measures}

Velocity measures were reduced to a three-component configuration with the components accounting for $55 \%, 24 \%$, and $6 \%$ of the total

Table 1

Selected anthropometric data and distances thrown.

\begin{tabular}{|c|c|c|c|c|c|c|c|c|c|}
\hline \multirow[t]{2}{*}{ Subject } & \multirow{2}{*}{$\begin{array}{l}\text { Height } \\
\text { (cm) }\end{array}$} & \multirow{2}{*}{$\begin{array}{l}\text { Mass } \\
(\mathrm{kg})\end{array}$} & \multirow{2}{*}{$\begin{array}{l}\text { Axillary } \\
\text { circ. } \\
\text { (cm) }\end{array}$} & \multirow{2}{*}{$\begin{array}{l}\text { Forearm } \\
\text { circ. } \\
(\mathrm{cm})\end{array}$} & \multirow{2}{*}{$\begin{array}{l}\text { Wrist } \\
\text { circ. } \\
\text { (cm) }\end{array}$} & \multicolumn{3}{|c|}{ Distance $^{\mathrm{a}}(\mathrm{m})$} & \multirow{2}{*}{$\begin{array}{l}\text { Skill } \\
\text { rank }\end{array}$} \\
\hline & & & & & & $\overline{\mathbf{L}}$ & $\mathbf{M}$ & $\mathrm{H}$ & \\
\hline 1 & 186.2 & 80.0 & 30.3 & 29.1 & 16.8 & 54.7 & 36.9 & 28.0 & 2 \\
\hline 2 & 186.3 & 79.3 & 30.9 & 29.3 & 17.4 & 74.7 & 42.6 & 29.9 & 1 \\
\hline 3 & 174.4 & 74.2 & 29.9 & 28.8 & 17.4 & 52.0 & 29.2 & 19.3 & 9 \\
\hline 4 & 174.9 & 89.4 & 36.4 & 29.0 & 18.4 & 39.4 & 24.4 & 16.6 & 12 \\
\hline 5 & 177.6 & 75.6 & 30.5 & 28.7 & 17.1 & 50.2 & $\div 2$ & 25.3 & 8 \\
\hline 6 & 177.6 & 75.7 & 33.6 & 31.1 & 17.5 & 59.8 & 28.9 & 22.0 & 6 \\
\hline 7 & 176.6 & 59.1 & 26.4 & 26.5 & 17.4 & 58.3 & 29.4 & 20.9 & 7 \\
\hline 8 & 184.3 & 66.0 & 28.8 & 26.6 & 17.3 & 61.0 & 30.0 & 21.6 & 3 \\
\hline$y$ & 178.6 & 60.0 & 29.2 & 26.9 & 16.6 & 42.4 & 28.5 & 20.2 & 11 \\
\hline 10 & 179.1 & 66.3 & 30.6 & 27.3 & 16.1 & 547 & 33.4 & 24.1 & 4 \\
\hline 11 & 180.5 & 79.3 & 32.7 & 29.8 & 17.1 & 56.8 & 31.5 & 22.7 & 5 \\
\hline 12 & 172.0 & 71.4 & 30.9 & 26.9 & 17.3 & 49.0 & 30.4 & 21.0 & 9 \\
\hline $\bar{x}$ & 179.0 & 73.0 & 30.9 & 28.3 & 17.2 & 54.4 & 31.5 & 22.6 & \\
\hline$S D$ & 4.6 & 8.9 & 2.5 & 0.2 & 0.6 & 9.2 & 4.6 & 3.7 & \\
\hline
\end{tabular}

a $L, M$, and $H$ repiesent the trials with the light, medium and heavy balls, respectively.

b The averages of each subject's three throws were ranked from highest to lowest. 
Table 2

Component loadings for the velocity measures mode.

\begin{tabular}{|c|c|c|c|}
\hline \multirow[t]{2}{*}{ Velocity measure } & \multicolumn{3}{|c|}{ Component loadings $(\times 100)^{a}$} \\
\hline & $\overline{\mathrm{Cl}}$ & $\mathrm{C2}$ & $\mathrm{C3}$ \\
\hline $\begin{array}{l}\text { Forearm }(X)^{b} \\
\text { Upper arm }(X) \\
\text { Hand }(X)\end{array}$ & $\left.\begin{array}{l}-53 \\
-40 \\
-26\end{array}\right)$ & $\begin{array}{r}3 \\
-46 \\
47\end{array}$ & $\begin{array}{r}24 \\
28 \\
-70\end{array}$ \\
\hline $\begin{array}{l}\text { Forearm }(Y)^{c} \\
\text { Upper } \operatorname{arm}(Y) \\
\text { Hand }(Y)\end{array}$ & $\left.\begin{array}{l}53 \\
36 \\
30\end{array}\right\}$ & $\begin{array}{r}-1 \\
-54 \\
51\end{array}$ & $\begin{array}{r}-38 \\
48\end{array}$ \\
\hline
\end{tabular}

Component contrasts

$\bar{X}$ vs. $\mathbf{Y}$

Prox. vs. Dist.

$\mathrm{U} / \operatorname{Ar}(\mathrm{X})$ ivs. $\mathrm{U} / \mathrm{Arm}(\mathrm{Y})$

Hand $(\mathrm{Y})$ ivs. : Hand $(\mathrm{X})$

Total explained

variation (percent)

55

24

6

Arrows indicate contrasts.

b $\mathrm{X}$ indicates the horizontal velocity (positive values: forward; negative values: back).

c $\mathrm{Y}$ indicates vertical velocity (positive values: up; negative values: down).

variance. The component loadings for each of the segment velocity measures are displayed in table 2. The first component distinguishes between horizontal $(X)$ and vertical $(Y)$ velocity with the component loadings for the horizontal velocity of opposite sign to those of the vertical velocity. This component accounts for most of the variance (55\%) of these data and indicates the average relationship among the segment velocity measures across all time periods, subjects and ball weights. This component was interpreted as directional velocity. In gencial, when the segments move forward (in the direction of the throw) they also tend to move down, and when they move backward they also move up.

The second component marks the contrast between proximal and distal segment velocity measures. Thus, the loadings on this component for the hand and arm are high but of opposite sign. Not unexpectedly, the forearm velocity measures lie between those of the hand and the arm. The relationship reflected by this component was interpreted as proximal versus distal velocity. Indeed, when the hand is moving in the positive directions (forward or up), the arm moves in the negative directions (backward or down), and vice versa. 
A third component, which accounts for a small percentage of the total variance $(6 \%)$, reflects a relationship among the arm and hand segment velocities which, as will become apparent later, occurs during the final few time samples of the throw. Specifically, movement of the hand forward and the arm up is contrasted by hand movement up and arm movement forward. These movements are characteristic of the final release action when all segments move forward and upward to whip the forearm and hand through to release.

The relationships indicated by the component loadings characterize some phase of the throwing action. The component solution reflects the dominant relationships among the arm segments during the entire throw. Because most of the time of the throw is spent in preparatory movements (windup) and relatively little time is spent in the release phase, the first component tends to model the early aspects, while the higher order components, two and three, tend to characterize small sections of the throw, and less dominant segmental relationships.

\section{Time periods}

Four components were extracted for the time periods mode and these accounted for $32 \%, 23 \%, 17 \%$, and $3 \%$, respectively, of the total variance. Fig. 2a shows the loadings of the 28 time periods plotted in the space of component one (C1) and two (C2). Stick figures, which are superimposed on this figure, show approximate body positions at the six time phases. These are only included to help the reader picture the movement sequence and illustrate the postures which are indicative of the six phases of the movement. The elliptical shapes represent the throwing limb while the dotted lines indicate the position of the non-dominant limb. The inclination of the trunk is shown and the direction of the throw is indicated by the way in which the figure is facing.

Component one is loaded most heavily by those time periods in phases III, IV, and VI. That is, the projections of the points in time on the $\mathrm{C} 1$ axis, during these phases, fall at large distances from the origin. For example, the projection of time period 28 has been drawn in as a dashed line, and intersects the $\mathrm{Cl}$ axis a long way from the origin. Similarly, those time periods which characterize phases I and II are weighted most heavily on component two. From this figure it can be seen that most of the time of the throw is spent in preparatory 


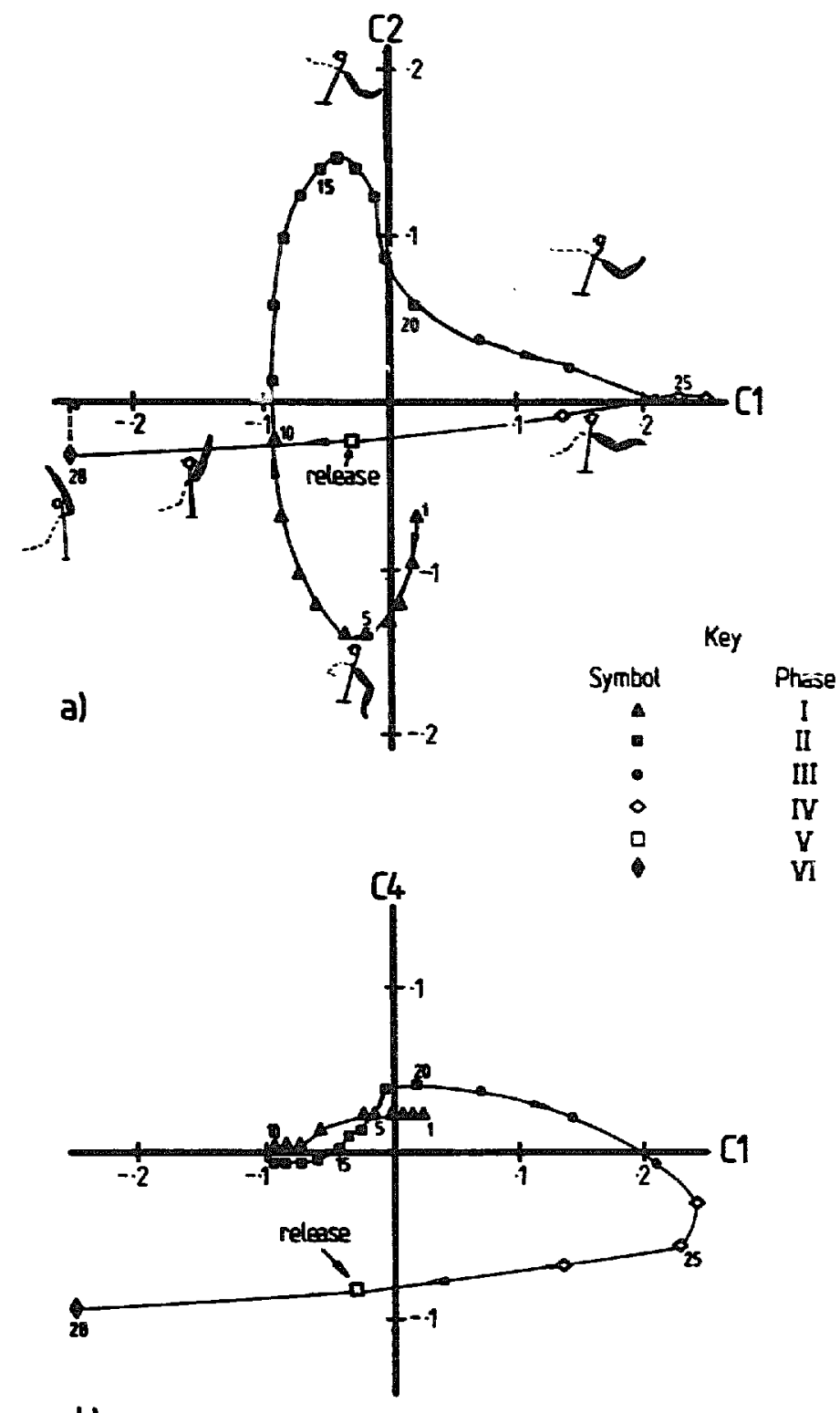

b)

Fig. 2. An illustration of the component solution of the time periods mode, Components 1 and 2 are plotted in (a) and components 1 and 3 are plotted in (b). The stick figures indicate approximate body positions in the various phases.

movements (phases I, II, and III include 23 of the 28 time samples) while relatively little time is needed to complete the whip-like action of the arm segments during release. That is, the greatest amount of variability of these data lies at the time samples $23,24,25$ and 28 .

Fig. $2 b$ shows a similar plot to fig. $2 a$ except that the fourth component is plotted on the ordinate. This component illustrates the 
distinction between the preparatory phases and release phase of the throw. It can be seen that the projections of the time samples of phases I-III on the $\mathrm{C} 4$ axis cluster together above the $\mathrm{Cl}$ axis while the projections of the time periods of the other three phases cluster together below the $\mathrm{C} 1$ axis. Component 3 (not illustrated) primarily distinguishes between the frame after release (28) and the other time periods.

The above results, combined with the information about the velocity measures mode, indicate that relationships among limb segnents that are established in the three early phases are different to those which characterize the last three phases. Thus, as noted earlier, in the last few time samples, in particular phases IV and V, all of the arm segments tend to be moving up and forward. However, at the last time sample, phase VI, the recoil action of the arm and the flick of the wrist and fingers are apparent as the segment velocity relationships change.

\section{Experimental units}

In the experimental unit space, it took three components to extract $75 \%$ of the total variance, with the component eigenvalues accounting for $39 \%, 23 \%$, and $13 \%$ respectively. The variance, spread across the person components, indicates that although individuals went through similar arm segment velocity relationships, they did so at different phases of the throw. Individuals within the same groups display patterns of velocities which are linear combinations of each other.

Fig. 3 shows the subjects' scores for all three trials on the first and second components. The first component $(\mathrm{C} 1)$ is loaded positively by all subjects across all their throws. This component seems to indicate a relationship which is common to all the throwers. The second component begins to distinguish between throwing styles. In fact, this component may be deemed to correspond to technique or skill level. The two best throwers of the group (S1 and S2), as indicated by experience in throwing sports and distances thrown, along with S7, a subject who displayed good technique, are clustered together. Their scores on component 2 are opposite to those of subjects 3,5 , and 11 who grouped together but included two of the poorest throwers (S3 and S5) of this sample of athletes.

While the extent of variation for load was not as marked as the individual differences among throwers, an effect of the different 


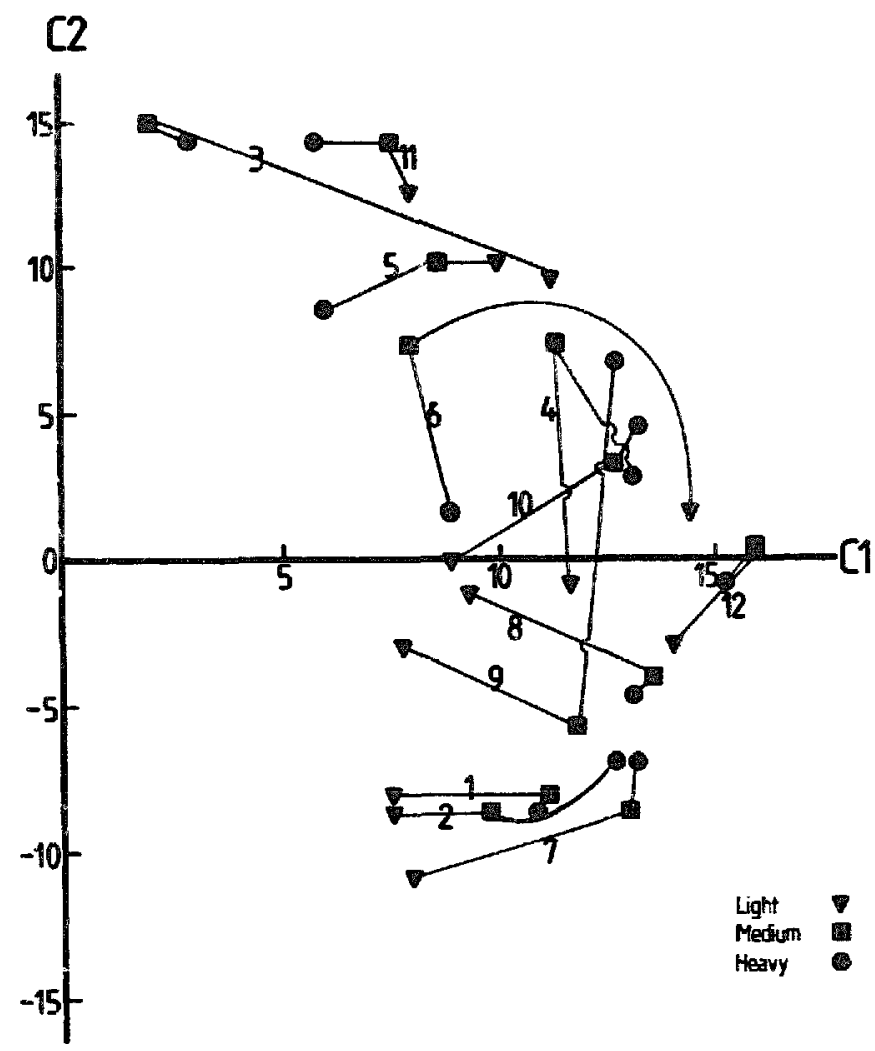

Fig. 3. The component space (components 1 and 2) of the experimental units mode. The highly-skilled subjects have negative scores on component 2 whereas the less-skilled subjects have positive scores.

weighted softballs was evident on component 3 of the experimental units mode. Fig. 4 shows components 2 and 3 plotted on the abscissae and ordinate respectively. This figure illustrates a general relationship between the score on component 3 and the weight of the ball. That is, as the weight of the ball increased, so too did the score on component 3. This relationship is more noticeable for the less-skilled subjects (e.g., S6, S8, S9, S11, and S12) than for the highly-skilled athletes (e.g., S1, S2, and S7). From these data there does seem to be a slight modification of technique due to the increased weight of the ball. Note, however, that the load increase is a within-subject phenomenon and there are large between-subject variations which are less easily explained.

Each experimental unit (subject $\times$ condition) can be seen as a linear combination of the components identified by the three-mode analysis. The following examples help to illustrate this point. Without going into the technical detail, the data for the light condition of throwing for 


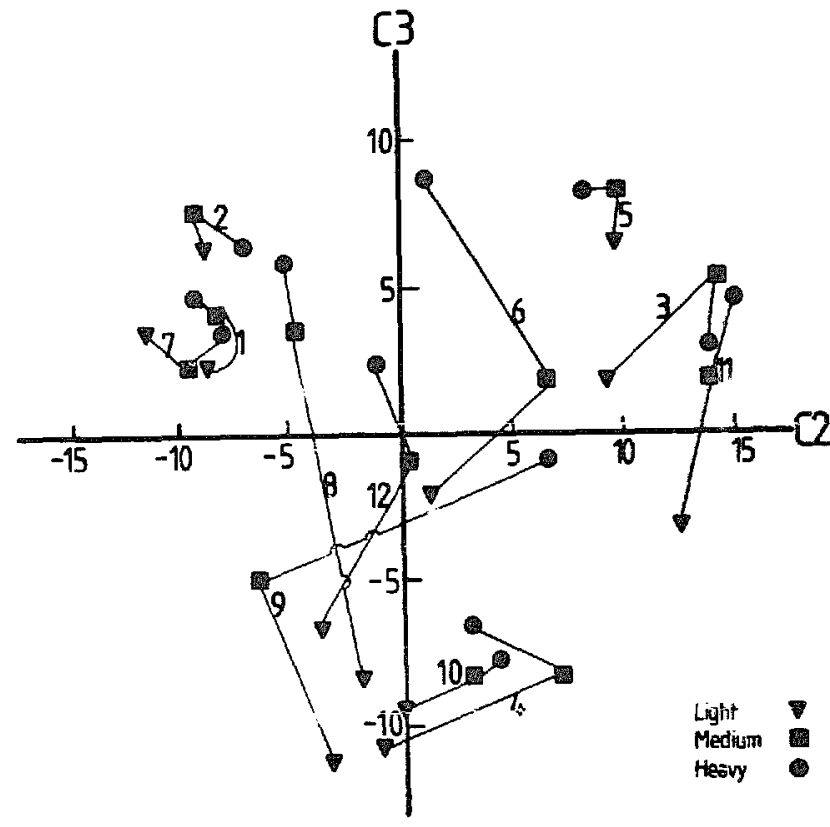

Fig. 4. The component space (components 2 and 3 ) of the experimental units mode. The influence of ball weight is seen as the scores on component 3 for some subjects increases.

subjects 5 and 7 can be reconstructed via the three-mode model using equations such as the ones below, but with differing outcomes:

S5(light $)=0.12 d_{1}-0.18 d_{2}+0.18 d_{3}$,

$\mathrm{S} 7$ (light) $=0.17 d_{1}+0.20 d_{2}+0.05 d_{3}$

where, $d_{r}, r=1,2,3$ are similar to component scores for subject component $r$ (for mathematical details see Kroonenberg 1983: 166).

Both subjects exhibit a technique which contains the general characteristics of a throwing motion (i.e., positive weight on component 1) but the difference in their technique is marked on the second component (skill level). The two weights are of opposite sign indicating that the subjects are at opposite ends of the skill level spectrum. Perusal of the distances that they threw the balls and their anthropometric development (table 1) reveals that S2 had the best throwing record and S3 had the worst performances while their muscular development, as indicated by segment girth measurements, were similar. 
A similar technique can be used to illustrate the influence of load on throwing patterns. S12's data provide a good example. The component loadings for his three throws are shown below:

$\mathrm{S} 12($ Light $)=0.22 d_{1}-0.07 d_{2}-0.18 d_{3}$.

$\mathrm{S} 12($ Medium $)=0.25 d_{1}+0.01 d_{2}-0.02 d_{3}$.

$\operatorname{S} 12($ Heavy $)=0.23 d_{1}-0.02 d_{2}+0.07 d_{3}$.

This subject's scores on the first two components changed little in response to the increased weight of the ball. However, there is a systematic within-subject change from negative scores to positive scores on the third component as the weight of the ball progresses from light to heavy.

\section{Mode interactions}

Having obtained the measures and time periods solutions, the interaction of these modes can ba obtained by projecting the velocity measures vectors and those of the time periods into one space (the joint space) to ascertain the particulars of the sequential relationships of the segment involvement in the throw. For each person component there is a separate joint space derived using the slice of the core matrix associated with that component. In the analysis of the data of this study, there emerged three general patterns associated with each of three person components. The characteristics of the first two of these components will be described in detail since they account for $82 \%$ of the fitted variance while the third will only be described in a cursory manner.

Since three-mode analysis is a components solution, the person components are idealized forms and do not necessarily relate to one particular athlete. The athletes can be seen, therefore, as a combination of the three person components with their distinctive throwing styles determined by specific loadings on the person components identified by the analysis.

\section{Segment involvement}

The relative importance of any arm segment velocity at any point in time during the throw can be assessed using the joint plot information. 
Table 3

Inner products of the velocity measures and the time periods at selected times during the throw for the three person components.

\begin{tabular}{|c|c|c|c|c|c|c|c|}
\hline \multirow[t]{2}{*}{ Time } & \multirow[t]{2}{*}{ Phase } & \multicolumn{2}{|l|}{ Hand } & \multicolumn{2}{|c|}{ Forearm } & \multicolumn{2}{|l|}{ Arm } \\
\hline & & $\overline{x^{a}}$ & $Y^{b}$ & $\bar{x}$ & $\mathbf{Y}$ & $\mathbf{X}$ & $\mathbf{Y}$ \\
\hline & & \multicolumn{4}{|c|}{ Person component 1} & & \\
\hline 1 & I & 1 & -2 & 4 & -4 & 4 & -2 \\
\hline 6 & 1 & 4 & -2 & 4 & -4 & 1 & -3 \\
\hline 12 & II & 0 & 0 & -4 & 3 & -3 & 4 \\
\hline 18 & II & -3 & -2 & -3 & 3 & 0 & 5 \\
\hline 23 & III & 2 & 7 & -1 & 2 & -5 & -4 \\
\hline 25 & IV & 1 & 11 & -2 & 4 & -8 & -6 \\
\hline 27 & $\mathrm{~V}$ & -5 & 1 & 3 & -1 & 4 & -3 \\
\hline \multirow[t]{2}{*}{28} & VI & -10 & -9 & 9 & -6 & 16 & 1 \\
\hline & & \multicolumn{3}{|c|}{ Person component 2} & & & \\
\hline 1 & 1 & 3 & 0 & 1 & -2 & -1 & -2 \\
\hline 6 & 1 & -2 & 1 & -4 & 4 & -3 & 4 \\
\hline 12 & II & -3 & 0 & -3 & 3 & 0 & 3 \\
\hline 18 & II & 1 & -1 & 4 & -3 & 3 & -3 \\
\hline 23 & III & 6 & 0 & 4 & -4 & -1 & -5 \\
\hline 25 & IV & 5 & 1 & 3 & -3 & -1 & -5 \\
\hline 27 & V & -1 & -1 & 1 & -1 & 1 & 0 \\
\hline \multirow[t]{2}{*}{26} & VI & -4 & -3 & 1 & -1 & 4 & 2 \\
\hline & & \multicolumn{3}{|c|}{ Person component 3} & & & \\
\hline 23 & III & -1 & 5 & -4 & 4 & -5 & 0 \\
\hline 27 & V & 0 & -2 & 0 & -1 & -2 & 1 \\
\hline 28 & VI & 2 & -7 & 3 & -5 & 5 & 1 \\
\hline
\end{tabular}

a $X$ indicates the horizontal velocity.

${ }^{\mathrm{h}} \mathrm{Y}$ indicates vertical velocity.

Those velocity measures with the largest projections onto the vectors of the time periods have the greatest influence at that time period. Numerically this notion can be expressed as the inner product of the time periods and velocity measures vectors.

The inner product for each of the velocity measures with the time period vectors, at selected time periods are displayed in table 3. Data from all three person components are included but not all time periods are displayed to maintain inspectability of the table. The time periods have been sampled in order to give a complete picture of the influential velocity measures at various phases of the throw. The sign of the inner 
product indicates the direction of movement of the segment centers of mass with positive indicating movement which is forward or up and negative reflecting movement that is backward or down.

From table 3 a pattern of segment involvement and sequencing can be determined. For person component 1 , shared by all subjects, the early parts of the movement are predominantly influenced by the forward and upward changes in velocity of the forearm and the arm. The next phase of the movement, time periods 12-18 particularly, are dominated by the upward and backward velocity of the forearm and the upward movements of the arm. The late and release phases of the movement (24-27) sees the first time that the changes in velocity of the hand are important. It moves forward and up while the arm moves backward and down. This interaction suggests a blocking action where the velocity of the proximal segment is decreased to allow the distal segments (i.e., the forearm and hand) to whip through and make a contribution to the action. The final time sample, one frame after release, sees the hand moving down and backward as the arm tends to move forward in the direction of the throw.

The interesting feature of this style is that the forearm and arm tend to move as a single unit. That is, the signs of the inner products, particularly early in the throw, are the same. The movement of this linked unit contrasts the movements of the hand: when the linked unit is moving forward or up (backward or down) the hand is moving backward or down (forward or up). This type of interaction among arm segments is similar to the technique advocated by Ariel (1976: 45) for 'well executed throws'. The timing of segment involvement, with the proximal segments dominating first, followed by the distal segments, lends support to the timing aspect of the summation of speed principle. It should be noted, however, that this principle states that the order of segment involvement should progress sequentially, segment by segment, from proximal to distal. The data from the present study do not indicate such a ricidly defined sequence of involvement.

All athletes have positive loadings on person component 1 for all their trials. This general style is indicative of this group of subjects and characterizes the 'throwing action'. Superimposed on this component is the influence of the second person component, associated with skill level of the subjects.

The highly-skilled athletes load negatively on this component whereas the less-skilful subjects have positive loadings. The time periods when 
the inner products have high magnitudes are phases II and IV. These phases, respectively, correspond to the times when the arm begins its upward motion during the windup and when it begins accelerating towards the target for release.

During phase II, the dominant velocity measures of the highly-skilled subjects are indicated below as a contrast between movements of some segments down and others forward.
Arm down )
Forearm down)
contrasted by
(Hand forward
(Forearm forward

The opposite segment configuration is true for the less-skilled subjects indicating that they move through a smaller range of motion than their skilful counterparts. The important relationships among the arm segments for the less-skilled athletes is summarized as,
Arm up
Forearm up )
(Hand back
contrasted by
(Forearm back

This set of contrasting relationships between the highly- and less-skilled subjects reverses during phase IV of the throw. At this point in time, the following picture reflects the technique of the highly-skilled subjects.

Hand back )

Forearm back )

(Arm up

contrasted hy

(Forearm up

The opposite situation is true for the less-skilled subjects. For the skilful athletes, the relationships indicated by the three-mode analysis give credence to the notion that when the arm and forearm begin moving up to commence the whip-like release action, the hand and the forearm move backward to pre-stretch the muscles that cross the wrist and elbow joints. This combination of events would place the muscles in a favourable position to produce maximum force (see e.g., Gollhofer et al. 1987, on stretch-shortening cycles). The less-skilled subjects show a pattern of movement in which the hand and fortaim segments begin 
to move toward the target 'too early', thereby losing the effect of pre-stretching the muscles.

The third person component highlights the differential influence of the load condition (ball mass) on throwing technique. It is superimposed onto the combined effects of person components 1 and 2. The influence of this component can be discussed in terms of the general throwing pattern and skill level. The times at which the velocity measures have an important effect, with respect to the third person component are displayed in table 3 . The segment relationships and contrasts for the heavy condition are summarized below:

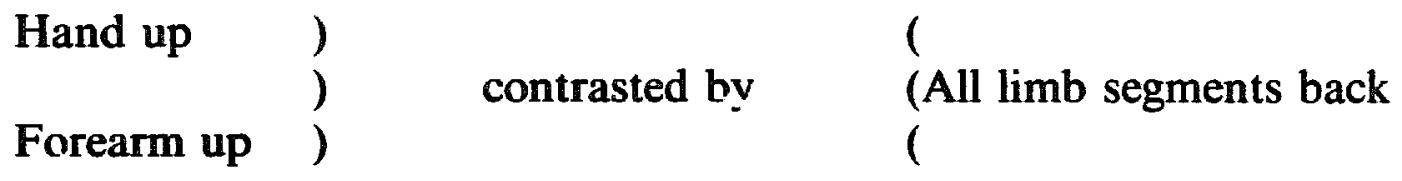

The opposite set of contrast and relationships would be indicative of the light condition. The effect of ball mass on the style of the highlyskilled subjects and two of the less-skilled subjects (S3 and S5) is minimal whereas the effect for some of the other subjects (S6, S8, S9, and S12) is marked. Interestingly, however, the increased weight of the ball appears to force a modification to the technique of these subjects which brings them in line with the actions adopted by the highly-skilled subjects. It is apparent that the additional load induces a timing pattern which allows the proximal segments to contribute first, followed by the distal segments. Thus, the 'mistiming' of segment involvement by some of the subjects is not evident, to the same extent, under the heavy condition as it is in the light condition.

\section{Summary}

Although there are wide individual differences in throwing style, the i ilationships of the arm segment velocities for different people and ball weights are summarized by a three-mode analysis. Three individual difference components are discernible in terms of the velocity changes over the throwing action. One of these is characteristic of all subjec : and appears to relate to a general overarm throwing motion. The second component relates to the influence of skill level whereas the third component reflects the effect of the varying ball weights on some 
of the athletes' throwing styles. There appears to be important time phase contrasts in phase I versus phase III and phase II versus phases IV and V. That is, segment velocity relationships established in phase I are countered in phase III and those established in phase II are countered in phases IV and V. Thus, the contrasts are critical in all motions; the segment velocity relationships of each phase constitute the 'throwing style'.

The segment involvement in the throw gives support for the timing aspect of the summation of speed principle. In the early and windup phases of the throw, the changes in velocity of the proximal segments, the arm and forearm, account for the largest percentage of the explained variance. It is not until the final whip-like action of release that the changes in velocity of the hand segment begin to become important. This type of sequential involvement of body segments has been advocated by a number of authors (Ariel 1976; Dyson 1973; Milburn 1982) when maximum speed of the distal link in a kinematic chain of segments is desired.

Skill level was distinguishable in terms of person component 2. It was evident that the highly-skilled subjects were able to move the arm segments through a greater range of movement and into more extreme positions than their lesser-skilled counterparts. This ability probably allowed pre-stretching of the muscles prior to the concentric contraction required to propel the ball through to release. Gregor et al. (1987) demonstrated, for a number of activities that pre-stretching of muscles allows the body to develop greater tension than in a non-stretched condition.

The highly-skilled subjects showed a pattern of segment involvement, particularly during time phases III and IV, in which the arm and forearm first accelerated toward the target, followed a little later in time, by the hand. The less-skilled subjects did not show this pattern. By contrast, the hand segment was moving toward the target in phase III, indicating a 'mistiming' of segment involvement.

There was a differential effect of ball weight on throwing performance. The technique of the highly-skilled athletes changed little in response to the altered load indicating a 'programmed', action. However, some subjects changed their style considerably under the influence of increased load. Kelso (cf. 1981) has argued that connotations of a 'program' are best avoided by describing physical action in terms of a dynamic, biological language. Priority by this account is given to 
the systematic relations among variables, the synergies, collectives, or coordinative structures (Kugler et al. 1980). Load, under this paradigm, may be considered to be an order parameter, which when scaled beyond critical values forces abrupt changes to the behavior of the system.

When thinking in terms of an action which differs over individuals, the notion of an 'ideal' is softened by the recognition of different possible relationships to accomplish the same end. However, the search for general principles of movement should not overlook the specificity of such principles. Putnam (1980), for example, found that the summation of speed principle held for the kick but not for the dismount movement, and that the transfer of angular momentum principle held for the dismount movement but not for the kick. In this study, three individual difference components were identified. While some subjects clearly had similar loadings on these components, there were distinct differences among them too. Thus, when individuals are free to select an approach to a task the 'solutions' can look and be quite diverse.

Methodologies such as three-mode principal component analysis (see e.g., Law et al. (1984) for examples of other multivariate multi-mode procedures) help disentangle the individual differences in human action and assist in the identification of those nomothetic generalizations which hold for empirical data on movement. Clearly individuals differ in the way that they carry out the task to 'throw as far as possible'. A central question is, "Can these individual differences be adequately described by quantitative differences of the dynamic parameters of the system (e.g., mass, stiffness, and damping) or are they qualitatively different, thus requiring a mediary or transiation mechanism (motor schema) to account for systemic variations?' Perhaps by understanding more about the range and the possibilities of individual differences, the design logic of the motor system can be better rationalized.

\section{Appendix}

Suppose, for the moment, a researcher measured the velocity of the three segments of the upper limb at the moment of release as a number of subjects threw a softball. This set of data has two modes, subjects and velocity scores. Rather than deal with such large numbers of velocity variables, a principai component analysis could be undertaken to reduce the size of the set and find the underlying or basic velocity variables. In addition to determining these underlying variables, the way in which each 
of the subjects 'loads' on them can be calculated. However, say the researcher now has data on the velocity of these body parts, not just at release, but through the entire throwing motion. The data set is now a three-mode one consisting of subjects, velocity measures and now, time. This data set could be arranged in a matrix $Z$ with general element $Z_{i j k}$. Such data sets are suitable for three-mode principal component analysis which Tucker (1966) defined as

$Z_{i, k}=\sum_{p=1}^{r} \sum_{q=1}^{Q} \sum_{r=1}^{R} a_{i p} b_{j q} c_{k r} g_{p q r}$

with terms as defined previously.

If $P, Q$ and $R$ the number of components of each mode are set equal to $I, J, K$ the number of observations of each of the corresponding modes then $\hat{Z}_{i j k}=Z_{i j k}$, where $Z_{i j k}$ is a general element of the observed data matrix $Z$. In practice the three-mode data matrix is not decomposed into all its components as one is only interested in those first few that account for the majority of the variance of the data. Thus, one seeks an approximate decomposition $\hat{Z}$ that is minimal according to a least squares loss function. That is, one solves for a $\hat{Z}$ such that

$\sum_{i=1}^{I} \sum_{j=1}^{J} \sum_{k=1}^{K}\left(\hat{Z}_{i j k}-Z_{i j k}\right)^{2}$

with $\hat{Z}_{i j k}$ as defined above, attains a minimum. This new matrix $\hat{Z}$ has the features that it reflects the general characteristics of $\mathcal{Z}$ but has substantially fewer rows (velocity measures), columns (time periods) and tubes (experimental units), as depicted in fig. 1 . Thus $\hat{Z}$ can be used to understand the essential information contained in the original data set, without the cumbersome dimensions of the original data set from which $\hat{Z}$ was derived.

\section{References}

Alexander, M.J. and J.B. Haddow, 1982. A kinematic analysis of an upper extremity ballistic skill: The windmill softball pitch. Canadian Journal of Applied Sports Science 7, 209-217.

Ariel, G., 1975. 'Computerized biomechanical analysis of human performance'. In: J.L. Bluestein (ed.), Mechanics and sports. New York: ASME.

Ariel, G., 1976. Computerized biomechanical analysis of throwers at the 1975 Olympic Javelin Camp. Track and Field Quarterly Review 76, 45-49.

Bunn, J.W., 1972. Scientific principles of coaching. Englewood Cliffs, NJ: Prentice-Hall.

Cavanagh, P.R. and J. Landa, 1976. A biomechanical analysis of the karate chop. Research Quarterly 47 610-618.

Davis, K. and B. Blanksby, 1976. The segmental components of fast bowling in cricket. Australian Journal of Health, Physical Education and Recreation (Supplement) 71, 6-8.

De Boor, C., 1978. 'A practical guide to splines'. In: F. John, L. Sirovich, J. LaSalle and G. Whitham (eds.), Applied mathematical sciences, Vol. 27. Berlin: Springer-Verlag. 
Dempster, W.T. and G. Gaughran, 1967. Properties of body segments based on size and weight. American Journal of Anatomy 120, 33-54.

Dyson, G., 1973. The mechanics of athletics (6th ed.). London: University of London Press.

Elliott, B., J.R. Grove, B. Gibson and B. Thurston, 1986. A three-dimensional analysis of the fastball and curveball pitches in baseball. International Journal of Sport Biomechanics 1 , 20-28.

Gollhofer, A., P.V. Komi, N. Fujitsuka and M. Miyashita, 1987. Fatigue during stretch-shortening cycle exercises. II: Changes in neuromuscular activation of human skeletal muscle. International Journal of Sports Medicine (Supplement) 8, 38-47.

Gregor, R.J., P.V. Komi and M. Jarvinen, 1987. Achilles tendon forces during cycling. International Journal of Sports Medicine (Supplement) 8, 9-14.

Harshman, R.A. and M.E. Lundy, 1984. 'Data preprocessing and the extended PARAFAC model'. In: H.G. Law, C.W. Snyder, Jr., J.A. Hattie and R.P. ilcDonald (eds.), Research methods for multimode data analysis. Naw York: Praeger.

Hatze, H., 1976. The complete optimization of a human motion. Mathematical Biosciences 28, 99-135.

Hoshikawa, T. and S. Toyoshima, 1976. 'Contribution of body segments to ball velocity during throwing with non-preferred hand'. In: P.V. Komi (ed.), Biomechanics V-B. Baltimore, MD: University Park Press.

Hudson, J.L., 1986. Coordination of segments in the vertical jump. Medicine and Science in Sport and Exercise 18, 242-251.

Kelso, J.A.S., 1981. 'Contrasting perspectives on order and regulation in movement'. In: J. long and A. Baddeley (eds.), Attention and performance, Vol. IX. Hillsdale, NJ: Erlbaum.

Koniar, M., 1973. 'The biomechanical studies on the superposition of angular speeds in joints of lower extremities of sportsmen'. In: S. Cerquiglini, A. Venerando and J. Wartenweiler (eds.), Biomechanics III. Basel: S. Karger.

Kroonenberg, P.M., 1983. Three-mode principal component analysis: Theory and applications. Leiden: DSWO Press.

Kroonenberg, P.M., 1984. 'Three-mode principal component analysis'. In: H.G. Law, C.W. Snyder, Jr., J.A. Hattie and R.P. McDonald (eds.), Research methods for multimode data analysis. New York: Praeger.

Kroonenberg, P.M. and P. Brouwer, 1985. User's guide to TUCKALS3 (Version 4.0). Department of Education, Rijksuniversiteit Leiden, The Netherlands.

Kroonenberg, P.M. and J. de Leeuw, 1980. Principal component analysis of three mode data by means of alternating least-squares algorithms. Psychometrika 45, 69-97.

Kugler, P.N., J.A.S. Kelso and M.T. Turvey, 1980. 'On the concept of coordinative structures as dissipative structures: I. Theoretical lizes of convergence'. In: G.E. Stelmach and J. Requin (eds.), Tutorials in motor behavior. Amsterdam: North-Holland.

Kunz, H., 1974. 'Effects of ball mass and movement pattern on release velocity in throwing'. In: R. Nelson and C. Morehouse (eds.), Biomechanics IV. Baltimore, MD: University Park Press.

Law, H.G., C.W. Snyder, Jr., J.A. Hattie and R.P. McDonald (eds.), 1984. Research methods for nultimode data analysis. New York: Praeger.

Lesh, M.D., J.M. Mansour and S.R. Simon, 1979. A gait analysis subsystem for smoothing and differentiation of human locomotion data. Journal of Biomechanical Engineering 101, 205-212.

McClellan, J.H., T.W. Parks and L.R. Rabiner, 1973. A computei program for designing optimum FIR linear phase digital filters. IEEE Transactions on Audio and Electroacoustics AU21. 506-526.

McCloy, C.H., 1960. 'The mechanical analysis of motor skills'. In: W.R. Johnson (ed.), Science and medicine of exercise and sports. New York: Harper and Row.

Milburn, P.D., 1982. Summation of segmental velocities in the golf swing. Medicine and Science in Sports and Exercise 14, 60-64. 
Neal. R.J. 1988. The influence of load on movement patterns. Unpublished Ph.D. thesis, University of Queensland.

Neal, R.J. and B.D. Wilson, 1985. 3D kinematics and kinetics of the golf swing. International Journal of Sport Biomechanics 1, 221-232.

Plagenhoef, S.G., 1971. Patterns of human motion: A cinematographical analysis. Englewood Cliffs, NJ: Prentice-Hall.

Putnam, C.A., 1980. Segment interaction in selected two-segment motions. Unpublished Ph.D. thesis, University of Iowa.

Roberts, E.M., R.F. Zernicke, Y. Youm and T.C. Huang, 1974. 'Kinetic parameters of kicking'. In: R. Nelson and C. Morehouse (eds.), Biomechanics IV. Baltimore, MD: University Park Press.

Snyder, C.W., Jr., 1988. 'Multimode factor analysis'. In: ' iNesselroade and R.B. Cattell (eds.), Handbook of multivariate experimental psychology (2nd ed.) New York: Plenum.

Ten Berge, J.M.F., J. de Leeuw and P.M. Kroonenberg, 1987. Srme additional results on principal component analysis of three-mode data by means of alternating least squares algorithms. Psychometrika 52, 183-191.

Toyoshima, S., T. Hoshikawa, M. Miyashita and T. Oguri, 1974. 'Contribution of the body parts to throwing performance'. In: R. Nelson and C. Morehouse (eds.), Biomechanics IV. Baltimore. MD: University Park Press.

Tucker, L.R., 1966. Some mathematical notes on three-mode factor analysis. Psychometrika 31, 279-311. 